\title{
Les macrophages cérébraux : rôle dans les remaniements morphologiques et la dégénérescence des neurones
}

Phénotype particulier de la microglie, les macrophages cérébraux sont physiologiquement présents dans le système nerveux immature et réapparaissent dans le parenchyme cérébral adulte lors d'affections inflammatoires ou neurodégénératives. Ils se distinguent des cellules microgliales ramifiées observées dans le tissu sain selon plusieurs critères structuraux ou fonctionnels, dont l'activité de phagocytose. Par leurs interactions avec les différents types de cellules nerveuses, les macrophages cérébraux sont impliqués dans des processus de dégradation ou de remodelage tissulaire. A l'échelle du neurone, des observations récentes montrent que le macrophage peut faire office de tueur, mais il peut aussi, dans d'autres conditions, favoriser la survie des cellules ou des remaniements de leurs arborisations axonales.

\section{Michel Mallat Brigitte Chamak Clotilde Théry}

\section{ADRESSE}

Michel Mallat : chargé de recherche à l'Inserm. Brigitte Chamak: ingénieur de recherche $\dot{a}$ l'Inserm. Clotilde Théry : assistante à l'université Paris 6. Unité Inserm U. 114, Collège de France, 11, place Marcelin-Berthelot, 75231 Paris Cédex 05, France.
I nitialement décrite au début du siècle par Pio del Rio Hortega, la microglie est l'une des trois principales populations de cellules'gliales dans le système nerveux central. Son appellation reflète un caractère morphologique qui, dans le tissu adulte, la différencie des deux autres types gliaux que sont les astrocytes et les oligodendrocytes : un corps cellulaire de dimensions réduites mais muni de longs prolongements tortueux et ramifiés. Depuis quelques années, les histologistes ont recours à des anticorps spécifiques de marqueurs des lignages granulomonocytaires pour colorer sélectivement ces cellules microgliales, dites ramifiées, au sein du parenchyme
[1-3]. Elles sont, en règle générale, plus nombreuses dans la substance grise qu'au sein des faisceaux axonaux myélinisés (substance blanche). Chez la souris et selon les régions cérébrales, la proportion de cellules microgliales ramifiées varie entre 5 et $12 \%$ de la population cellulaire incluant la macroglie et les neurones [4].

Le terme de microglie est parfois étendu à des cellules localisées autour des capillaires et des petits vaisseaux. Dans des conditions physiologiques, ces cellules périvasculaires sont, au moins pour une part, isolées des cellules nerveuses par une expansion de la membrane basale bordant l'endothélium [5]. Chez le rat, comme chez 
d'autres espèces, elles expriment des antigènes macrophagiques autres que ceux détectés au niveau des cellules microgliales intraparenchymateuses [5,6] (Tableau I).

\section{Les phénotypes microgliaux dans le système nerveux immature}

L'origine embryologique des cellules microgliales fait encore l'objet de controverses. L'hypothèse la plus communément admise s'inspire du cas général des macrophages tissulaires: la microglie proviendrait de l'envahissement du tissu nerveux par des monocytes circulants [3, 7]. Dans des conditions physiologiques (respectant notamment l'intégrité des capillaires cérébraux), la pénétration de monocytes dans le cerveau de rat n'a pu être montrée qu'à des stades précoces de la vie postnatale (durant les cinq premiers jours suivant la naissance) [8]. Au-delà de cette période, la paroi des vaisseaux ou des capillaires cérébraux s'oppose au passage des monocytes ou, du moins, le limite fortement. Au sein même du parenchyme cérébral, quelques cellules microgliales sont déjà détectables à des stades précoces de la neurogenèse dans des structures télencéphaliques encore réduites à un neuroépithélium germinatif [9]. Sur un plan morphologique, des phénotypes microgliaux du cerveau immature sont évocateurs de monocytes ou de macrophages recrutés dans les tissus à l'occasion d'une réaction inflammatoire ; ils sont désignés sous le terme de microglie amiboïde ou de macrophages cérébraux $[1-3,7]$. Ces cellules s'accumulent transitoirement dans diverses régions cérébrales; leurs caractères structuraux ont été tout particulièrement étudiés au niveau de faisceaux axonaux tel le corps calleux où elles sont abondantes en période périnatale, avant la phase de constitution des gaines de myéline. Les macrophages cérébraux se distinguent aisément des cellules microgliales ramifiées du cerveau adulte par leur morphologie, leurs caractères ultrastructuraux (un corps cellulaire riche en organelles et comportant de nombreux lysosomes primaires ou secondaires témoignant d'une activité de phagocytose), et l'expression de divers marqueurs dont des activités enzymatiques intracellulaires de type estérase non spécifique, aryl sulfatase ou phosphatase acide, révélées in situ par des colorations cytochimiques. En outre, ces cellules peuvent se diviser $[7,10,11]$. Plusieurs observations histologiques suggèrent que les cellules microgliales ramifiées du cerveau adulte résultent de la diffusion et d'une différenciation progressive de macrophages cérébraux, comportant une involution du corps cellulaire et la perte des fonctions de phagocytose ainsi que l'émission de prolongements $[1,2,11]$ (figure 1, p. 770).

\section{Macrophages cérébraux et système nerveux central adulte}

Dans le cerveau adulte, des macrophages sont localisés dans les méninges bordant le tissu nerveux ou dans les formations vasculaires intraventriculaires au niveau desquelles se forme le liquide céphalo-rachidien [2]. Dans les conditions physiologiques, les macrophages cérébraux paraissent exclus du tissu nerveux proprement dit, à l'exception de quelques régions cérébrales comme la neurohypophyse [12] qui se distinguent par l'absence de barrière hématoencéphalique isolant le parenchyme du sang circulant. Cependant, les macrophages cérébraux sont fréquemment observés dans un tissu nerveux adulte lésé. En pathologie humaine, le recrutement intraparenchymateux ne se limite pas à des affections inflammatoires ou infectieuses telles que la sclérose en plaques [13] ou le SIDA (voir l'article de M. Tardieu et al. dans ce même numéro, p. 775) ; des phagocytes réapparaissent aussi en forte quantité à l'occasion d'affections dégénératives. Ainsi les macrophages s'accu-

\begin{tabular}{|c|c|c|c|c|c|}
\hline & $\begin{array}{l}\text { IMMUNOPHÉ } \\
\text { DE CELLULES MICRO }\end{array}$ & $\begin{array}{l}\text { Tableau I } \\
\text { TYPE DES DIFFÉR } \\
\text { IALES ET DES MC }\end{array}$ & $\begin{array}{l}\text { TES CLASSES } \\
\text { CYTES CHEZ }\end{array}$ & RAT & \\
\hline $\begin{array}{l}\text { Anticorps } \\
\text { monoclonal }\end{array}$ & Antigène & Microglie ramifiée & $\begin{array}{c}\text { Cellules } \\
\text { périvasculaires }\end{array}$ & $\begin{array}{l}\text { Macrophages } \\
\text { cérébraux }\end{array}$ & Monocytes \\
\hline $\begin{array}{l}\text { MRC OX-42 } \\
\text { W3/25 } \\
\text { ED1 } \\
\text { ED2 } \\
\text { KIM-2R }\end{array}$ & $\begin{array}{c}\text { Récepteur du complément } \\
\text { de type } 3 \\
\text { CD4 } \\
\text { Intracellulaire } \\
\text { Membranaire } \\
\text { Membranaire }\end{array}$ & $\begin{array}{l}+ \\
- \\
- \\
-\end{array}$ & $\begin{array}{l}- \\
+ \\
+ \\
+ \\
+\end{array}$ & $\begin{array}{l}+ \\
+ \\
+ \\
+ \\
+\end{array}$ & $\begin{array}{l}+ \\
+ \\
+ \\
- \\
-\end{array}$ \\
\hline
\end{tabular}

La microglie ramifiée correspond au phénotype détecté dans le parenchyme adulte en l'absence de lésions. Outre les cellules microgliales, I'anticorps MRC OX-42 marque les granulocytes, les monocytes et des macrophages tissulaires. W3/25 se lie à des thymocytes, aux lymphocytes $T$ auxiliaires (helper) et à des macrophages péritonéaux. Les anticorps monoclonaux ED1, ED2 et KIM-2R reconnaissent des antigènes biochimiquement indéterminés et sélectivement exprimés par des macrophages tissulaires [3, 5, 6]. Chez la souris, les cellules microgliales ramifiées du cerveau adulte expriment d'autres antigènes spécifiques des phagocytes mononuclés [3]. 


\section{RÉFÉRENCES}

1. Hume DA, Perry VH, Gordon S. Immunohistochemical localization of a macrophage-specific antigen in developing mouse retina : phagocytosis of dying neurons and differentiation of microglial cells to form a regular array in the plexiform layers. J Cell Biol 1983; 97 : 253-7.

2. Perry VH, Hume DA, Gordon S. Immunohistochemical localization of macrophages and microglia in the developing and adult mouse brain. Neuroscience $1985 ; 15$ : $313-26$.

3. Perry VH, Gordon S. Macrophages and microglia in the nervous system. Trends Neur Sci $1988 ; 11: 273-7$.

4. Lawson LJ, Perry VH, Gordon S. Heterogeneity in the distribution and morphology of microglia in the normal adult mouse brain. Neuroscience 1990 ; 39 : 151-71.

5. Graeber MB, Streit WJ, Kreutzberg GW. Identity of ED2-positive perivascular cells in rat brain. $J$ Neurosci Res 1989 ; 22 : $103-6$

6. Graeber MB, Streit WJ, Kiefer R, Schoen SW, Kreutzberg GW. New expression of myelomonocytic antigens by microglia and perivascular cells following lethal motor neuron injury. J Neuroimmunol 1990 ; $27: 121-32$.

7. Ling EA. The origin and nature of microglia. In : Fedoroff S, Hertz L, eds. Advances in Cellular Neurobiology, vol. 2. New York : Academic Press, 1981: 33-82.

8. Ling EA, Penney D, Leblond CP. Use of carbon labeling to demonstrate the role of blood monocytes as precursors of the ameboid cells in corpus callosum of post-natal rats. J Comp Neurol $1980 ; 193$ : 631-57.

9. Ashwell $\mathrm{K}$. The distribution of microglia and cell death in the fetal rat forebrain. Dev

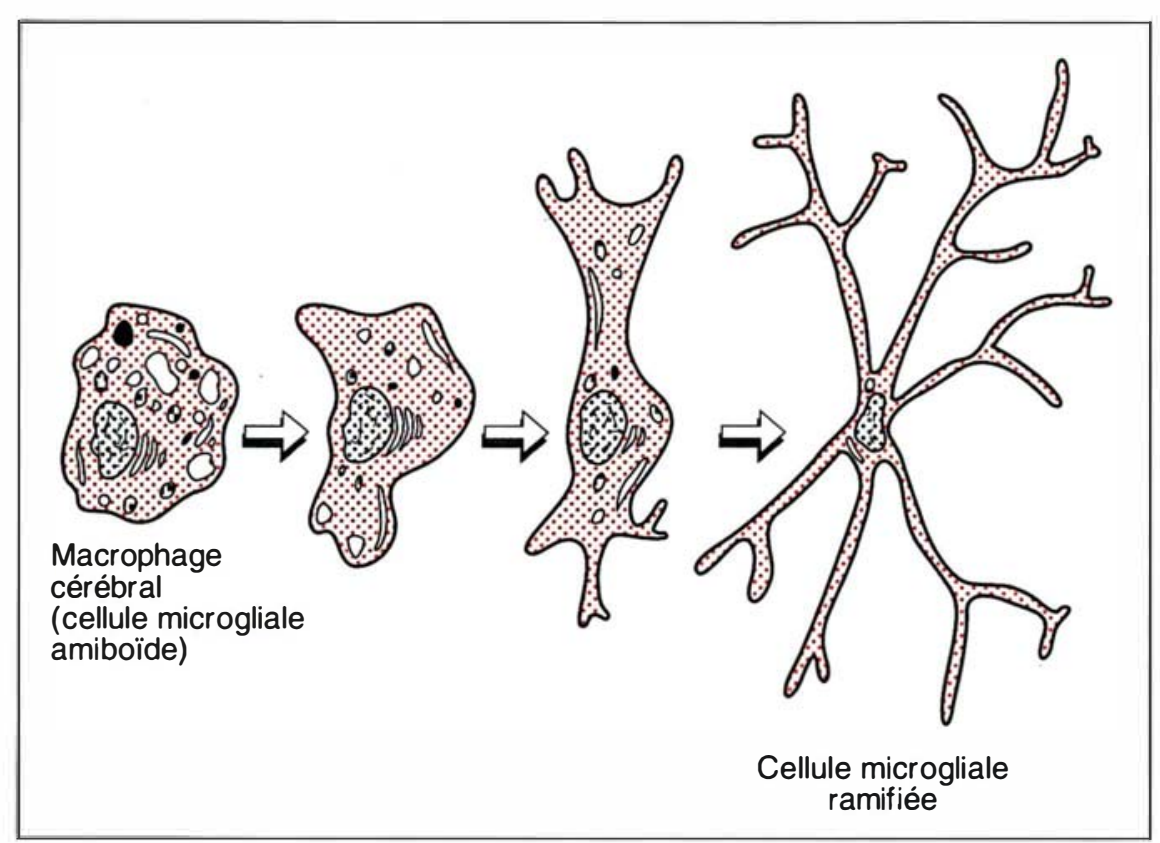

Figure 1. Transformation progressive de macrophages cérébraux en cellules microgliales ramifiées au cours du développement.

mulent-ils dans les régions cérébrales caractérisées par la mort ou des altérations neuronales lors des maladies de Parkinson ou d'Alzheimer [14]. De nombreux modèles expérimentaux animaux ont pour but d'analyser les modifications de la microglie secondaires à des altérations du tissu nerveux. Des travaux in vivo impliquent divers types de lésions d'origine ischémique, mécanique, toxique ou autoimmune [15-19]. La présence de phénotypes macrophagiques correspond aux phases initiales de la réponse microgliale, mais l'ampleur et la cinétique d'apparition de macrophages est largement déterminée par la nature de la lésion infligée. Une nécrose neuronale induite par injection locale d'une neurotoxine peut être suivie d'un recrutement rapide (en moins de 48 heures) et massif de phagocytes mononucléés dans la région où sont localisés des corps cellulaires en dégénérescence [18] (voir l'article de I. Dusart et al., dans ce même numéro, p. 790). A l'inverse, une dégénérescence de fibres nerveuses propagée après section du nerf optique (dégénérescence antérograde) provoque, à distance de la lésion initiale, une mobilisation macrophagique lente et modérée [17]. L'origine des macrophages recrutés au cours des lésions semble être diversifiée. Une infiltration de monocytes circulants, et leur transformation locale en macrophages, est claire dans de nombreux contextes impliquant une brèche ou des altérations des parois vasculaires [16, 18]. Cependant, l'origine exogène des macrophages recrutés a été contestée dans le cas de la dégénérescence de motoneurones du nerf facial du rat, induite par une axotomie combinée à l'injection d'une toxine végétale dans la portion extracérébrale des fibres nerveuses [20]. Dans ce modèle expérimental, des observations histologiques suggèrent que les macrophages cérébraux proviennent d'une différenciation des cellules périvasculaires et d'une transformation "inverse " de cellules microgliales ramifiées impliquant au niveau morphologique une hypertrophie du corps cellulaire associée à une rétraction des prolongements et des mitoses des cellules [6, 20]. En résumé, des monocytes circulants et des cellules microgliales ramifiées ou périvasculaires pourraient contribuer de manière combinée ou séparée au recrutement des macrophages. 


\section{Influence \\ des macrophages \\ sur la vitalité \\ et la morphologie \\ des neurones centraux}

Selon les contextes, la présence de macrophages cérébraux dans le parenchyme cérébral se traduit par d'importantes modifications au niveau des différents types de cellules nerveuses. Les macrophages peuvent être impliqués dans la destruction de gaines myéliniques des axones (formées par les prolongements d'oligodendrocytes) (voir l'article de $M$. Gumpel et al. dans ce même numéro, p. 782) ou dans la formation de cicatrices gliales post-lésionnelles [19, 21]. Ici, nous nous concentrerons plus particulièrement sur les interactions entre les macrophages cérébraux et les neurones centraux.

Nous avons déjà mentionné la présence de macrophages cérébraux dans le système nerveux immature. Leur localisation est compatible avec une influence sur la différenciation neuronale dès les phases les plus précoces de la neurogenèse. Cependant, les neuroanatomistes ont plus parti- culièrement relevé une corrélation dans plusieurs structures entre l'abondance transitoire de macrophages cérébraux et le déroulement d'événements régressifs physiologiques, dont la mort neuronale et l'élimination de collatérales axonales longues $[1,2,10,22]$. Ces deux événements contribuent massivement à l'ajustement des circuits neuronaux au cours de l'histogenèse cérébrale, leur ampleur et leur calendrier au cours du développement varient selon les types de neurones [23]. Durant ces phases de régression neuronale, l'activité de phagocytose des macrophages cérébraux ne semble pas strictement limitée à l'élimination de débris cellulaires. Un exemple de participation macrophagique active concerne la disparition de collatérales axonales survenant en l'absence de dégénérescence des neurones correspondants. Bien qu'un prolongement neuronal puisse éventuellement disparaître par simple rétraction, des observations ultrastructurales réalisées au niveau de la substance blanche du cortex cérébral de chatons montrent différents stades de séquestrations axonales dans des expansions cyto- plasmiques de macrophages cérébraux [24]. Dans le système nerveux adulte, des arguments histologiques analogues indiquent une amputation macrophagique de terminaisons axonales au niveau de la neurohypophyse. Rappelons que cette structure, dépourvue de barrière hématoencéphalique, héberge des cellules microgliales de phénotype macrophagique en contact avec les axones des neurones endocrines sécrétant de l'ocytocine ou de la vasopressine. L'élimination chronique des terminaisons axonales serait compensée par une capacité maintenue de régénération. Ce remodelage tissulaire permanent participerait à la régulation locale de la sécrétion neurohypophysaire [12].

Au-delà d'analyses histologiques pratiquées sur des tissus sains ou lésés, plusieurs protocoles expérimentaux, récemment mis en œuvre, visent à inhiber in situ les réactions microgliales secondaires à des lésions, en vue de préciser l'impact des macrophages cérébraux recrutés sur les cellules nerveuses [15, 18, 19]. En particulier, le retentissement sur les neurones a été plus spécifiquement étudié

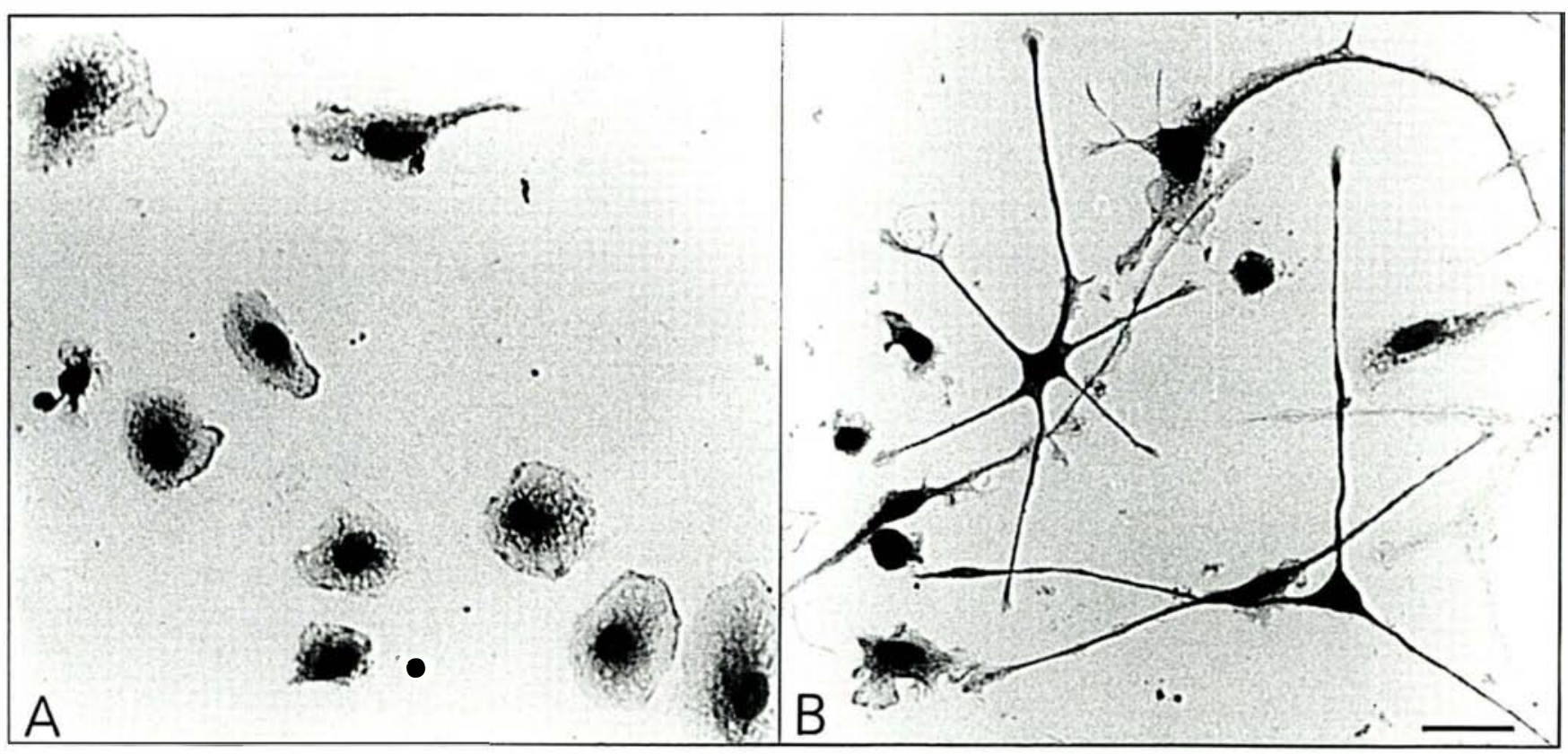

Figure 2. Morphologie de cellules microgliales issues du cortex cérébral d'embryons de rats (coloration au bleu de toluidine). Quarante-huit heures après leur isolement et selon les conditions de culture, les cellules conservent leur aspect de macrophage cérébral (A) ou présentent un phénotype évocateur de cellules microgliales ramifiées observées dans le cerveau adulte (B) (barre d'échelle: $50 \mu \mathrm{m}$ ) [42]. 


\section{RÉFÉRENCES}

10. Valentino R, Jones EG. Morphological and immunocytochemical identification of macrophages in the developing corpus callosum. Anal Embryol 1981 ; 163 : 157-72.

11. Imamoto K, Leblond CP. Radioautographic investigation of gliogenesis in the corpus callosum of young rats. II. Origin of microglial cells. J Comp Neurol $1978 ; 180$ : 134-64.

12. Pow DV, Perry VH, Morris JF, Gordon S. Microglia in the neurohypophysis associate with and endocytose terminal portions of neurosecretory neurons. Neuroscience $1989 ; 33: 567-78$.

13. Calder V, Owen S, Watson C, Feldmann M, Davison A. MS : a localized immune disease of the central nervous system. Immunol Today $1989 ; 3$ : 99-103.

14. McGeer PL, Itagaki S, McGeer EG. Expression of the histocompatibility glycoprotein HLA-DR in neurological disease. Acta Neuropathol 1988 ; 76 : 550-7.

15. Giulian DT, Robertson C. Inhibition of mononuclear phagocytes reduces ischemic injury in the spinal cord. Ann Neurol 1990 ; 27 : 33-42.

16. Imamoto $\mathrm{K}$, Leblond CP. Presence of labeled monocytes macrophages and microglia in a stab wound of the brain following an injection of bone marrow cells labeled with ${ }^{3} \mathrm{H}$-uridine into rats. J Comp Neurol 1977 ; 174 : 255-80.

17. Stoll G, Trapp BD, Griffin JW. Macrophage function during Wallerian degeneration of rat optic nerve : clearance of degenerating myelin and Ia expression. I Neurosci $1989 ; 9$ : 2327-35

18. Coffey PJ, Perry VH, Rawlins JNP. An investigation into the early stages of the inflammatory response following ibotenic acid-induced neuronal degeneration. Neuroscience $1990 ; 35$ : 121-32.

19. Huitinga I, Van Rooijen $N$, De Groot CJA, Uitdehaag MJ, Dijkstra D. Suppression of experimental allergic encephalomyelitis in Lewis rats after elimination of macrophages. J Exp Med $1990 ; 172$ : 1025-33

20. Streit WJ, Kreutzberg GW. The response of endogenous glial cells to motor neuron degeneration induced by toxic ricin. $J$ Comp Neurol $1988 ; 268$ : 248-63.

21. Giulian D, Chen J, Ingeman JE, 772 mononuclear phagocytes in wound healing after traumatic injury to adult mammalian brain. J Neurosci 1989 ; 9 : 4416-29.

22. Killackey HP. Glia and the elimination of transient cortical projections. Trend Neurol Sci $1984 ; 7$ : 225-6

23. Cowan WM, Fawcett JW, O'Learly DDM, Stanfield BB. Regressive events in neurogenesis. Science 1984; 225 : 1258-65.

24. Innocenti GM, Clarke S, Koppel $\mathrm{H}$. Transitory macrophages in the white matter of the developing visual cortex. II. Development and relations with axonal pathways. Dev Brain Res 1983 ; 11 : 55-66.

25. Giulian D, Baker TJ. Characterization of ameboid microglia isolated from developing mammalian brain. $J$ Neurosci $1986 ; 6$ : 2163-78.

26. Badwey JA, Karnovsky ML. Active oxygen species and the functions of phagocytic leukocytes. Ann Rev Biochem 1980 ; 49 : 695-726.

27. Halliwell B, Gutteridge JMC. Oxygen radicals and the nervous system. Trends Neur Sci $1985 ; 7$ : 22-7.

28. Frei K, Siepl C, Groscurth P, Bodmer S, Swerdel C, Fontana A. Antigen presentation and tumor cytotoxicity by interferon treated microglial cells. Eur J Immunol $1989 ; 19$ : 1271-8.

29. Selmaj KW, Raine CS. Tumor necrosis factor mediates myelin and oligodendrocyte damage in vitro. Ann Neurol 1988 ; $23: 339-46$.

30. Giulian D, Vaca K, Noonan A. Secretion of neurotoxins by mononuclear phagocytes infected with HIV-1. Science 1990 ; $250: 1593-6$

31. Brachet $P$. Le facteur de croissance nerveuse NGF : rôle dans la plasticité et la maintenance fonctionnelle de la cellule neuronale. médecine/sciences $1990 ; 6: 854-62$.

32. Hetier E, Ayala J, Denèfle $P$, et al. Brain macrophages synthesize interleukin 1 and interleukin 1 mRNA, in vitro. $J \mathrm{Neu}$ rosci Res 1988 ; 21 : 391-7.

33. Frei $\mathrm{K}$, Malipiero UV, Leist TP, Zinkernagel RM, Schwab ME, Fontana A. On the cellular source and function of interleukin 6 produced in the central nervous system in viral diseases. Eur I Immunol 1989 ; 19 : 689-94. dans un modèle expérimental fondé sur une ischémie segmentaire de la moelle épinière de lapins, induite par une occlusion prolongée ( $>20$ minutes) de l'aorte abdominale. Au niveau du tissu nerveux, cette manœuvre entraîne, dans un délai de 24 heures, une accumulation massive de macrophages suivie d'une mort des motoneurones. La mobilisation macrophagique peut être fortement réduite par des injections intraveineuses de colchicine et de chloroquine, pratiquées juste après l'occlusion artérielle, il s'ensuit à la fois une atteinte moins importante des fonctions motrices et une meilleure survie des motoneurones [15].

Ces analyses in vivo témoignent d'influences macrophagiques directes ou indirectes sur la morphologie et la vitalité neuronale. Des études ex vivo tentent de préciser ces interactions. Elles exploitent des techniques de purification de macrophages cérébraux à partir de cultures gliales primaires issues de cerveaux immatures [25]. Selon les conditions de culture, les cellules isolées conservent leurs caractères macrophagiques ou évoluent vers un phénotype évocateur de cellules microgliales ramifiées (figure 2, p. 771). Nous avons récemment étudié les effets des macrophages cérébraux sur des neurones embryonnaires dans des cultures de faible densité cellulaire optimalisant les observations morphologiques des cellules [43]. Les macrophages ont donc été rajoutés à des cultures de neurones en phase de croissance dans un milieu chimiquement défini, les deux populations cellulaires étant issues du cortex cérébral d'embryons de rat. Après une période de latence variable selon les proportions des deux types cellulaires (de l'ordre de 24 heures pour un rapport macrophages/neurones de 1/1), la présence de macrophages entraîne des altérations neuronales telles que des rétractions de prolongements ou des nécroses avec, parfois, phagocytose des résidus cellulaires (figure 3 ). La destruction des neurones met ici en jeu une propriété classique des phagocytes : la sécrétion d'anions superoxydes. Ce composé et ses dérivés en solution aqueuse $\left(\mathrm{H}_{2} \mathrm{O}_{2}, \mathrm{OH}^{*}\right)$ sont impliqués dans des activités bactéricides ou tumoricides des macropha- 


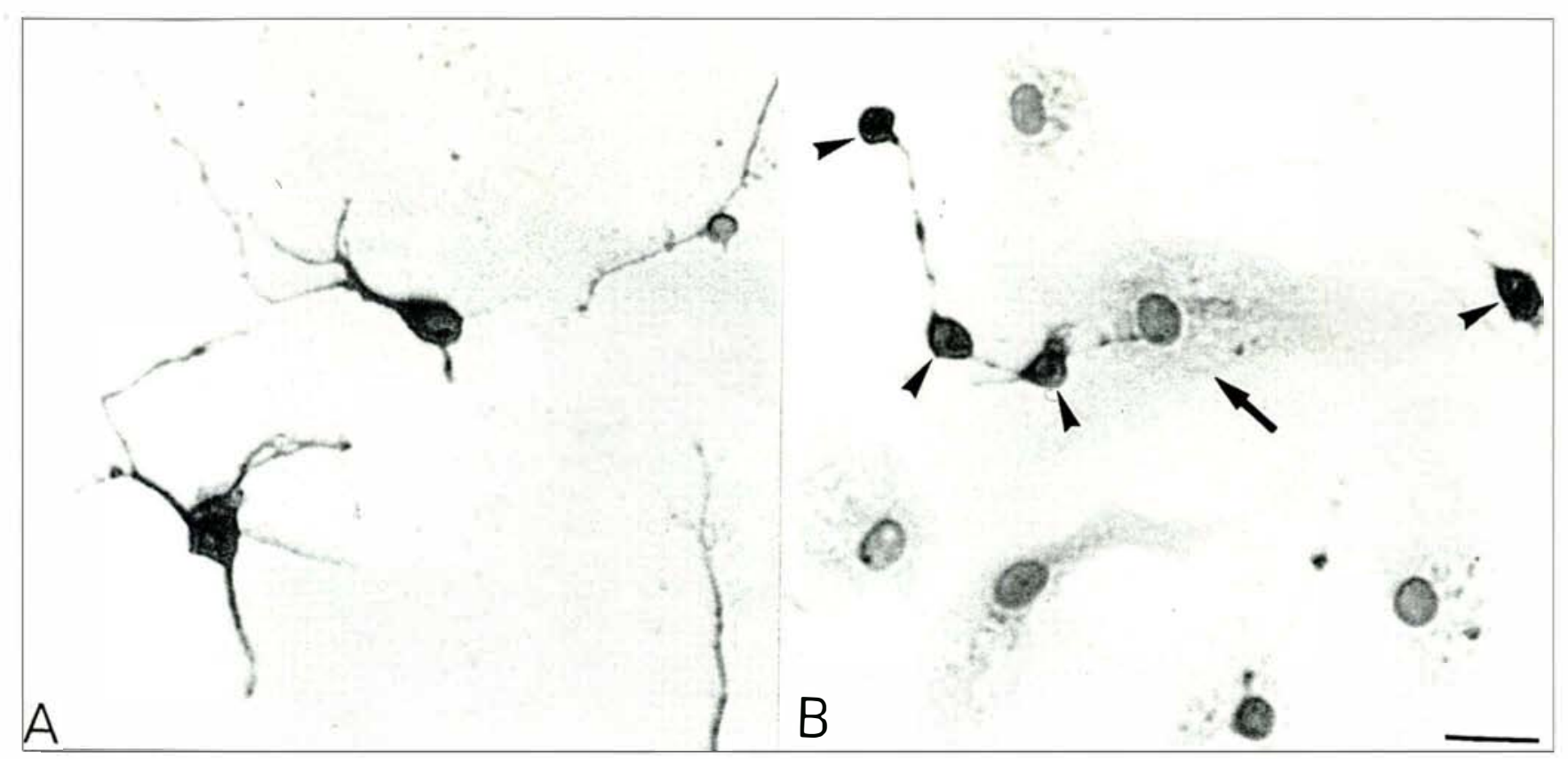

Figure 3. Influence des macrophages cérébraux sur la morphologie de neurones cultivés ex vivo. Les cellules sont issues du cortex cérébral d'embryons de rats. Les neurones ont été sélectivement marqués par immunocytochimie avec un anticorps anti-MAP2, 3 jours après leur mise en culture (coloration à la peroxydase). A : neurones cultivés en l'absence de macrophages cérébraux. B : neurones en présence de macrophages cérébraux ajoutés 48 heures avant la coloration. Les têtes de flèches indiquent les corps cellulaires neuronaux, une flèche désigne un macrophage dont une expansion entoure un neurone. Noter la réduction du nombre et de la longueur des prolongements neuronaux en présence des macrophages (barre d'échelle : $20 \mu \mathrm{m}$ ) [43].

ges tissulaires [26]. Leur nocivité varie selon les types de cellules exposées, elle est très marquée dans le tissu nerveux dont les membranes plasmiques, riches en acides gras insaturés, sont rapidement altérées par la propagation de peroxydations lipidiques [27]. Précisons que la production ex vivo de radicaux oxygénés par les macrophages cérébraux n'est pas constitutive mais qu'elle est induite par le contact avec les neurones selon un mécanisme qui reste à préciser.

Les radicaux libres oxygénés sont-ils les seuls vecteurs d'une neurotoxicité directe des macrophages cérébraux ? A l'image d'autres phagocytes mononucléés, les macrophages cérébraux peuvent sécréter du TNF $\alpha$, une cytokine toxique pour les oligodendrocytes qui assurent la myélinisation des fibres axonales [28, 29]. Cependant, les neurones cultivés ex vivo résistent à de fortes concentrations de cette molécule recombinante. Une autre option s'est récemment ouverte avec la mise en évidence de neurotoxines agissant par l'intermédiaire $\mathrm{m} / \mathrm{s} n^{\circ} 8$, vol. 7 , octobre 91 de récepteurs neuronaux de type glutamatergique et produites par des lignées monocytaires humaines en réponse à leur infection par HIV-1 [30]. On peut s'interroger sur l'éventuelle production de ces neurotoxines par des macrophages cérébraux dans divers contextes de neurolyse.

En dépit de la capacité des macrophages cérébraux à libérer des molécules cytotoxiques, on aurait tort de limiter leur rôle aux seuls événements neurorégressifs. La possibilité d'une action facilitatrice de ces cellules sur la croissance de prolongements neuronaux ou la survie de neurones peut, en effet, être envisagée par le biais d'une production locale de facteurs de croissance. A cet égard, l'exemple le plus étayé concerne le plus étudié des facteurs neurotrophiques: le NGF (nerve growth factor). Rappelons qu'entre autres activités biologiques le NGF est un facteur de croissance actif sur des neurones cholinergiques du SNC adulte ou immature (pour une revue et des références voir [31]). En particulier, l'injec- tion intracérébrale de NGF réduit la dégénérescence de neurones cholinergiques induite par une axotomie. Dans les conditions physiologiques (en l'absence de macrophages cérébraux), des neurones localisés dans l'hippocampe ou le néocortex synthétisent du NGF (ces deux régions cérébrales sont innervées par des neurones cholinergiques sensibles au NGF). Deux interleukines (1 et 6) produites par les macrophages cérébraux [32, 33] sont susceptibles de moduler les concentrations intracérébrales de NGF. L'interleukine 1 est biologiquement détectable dans diverses régions cérébrales lors d'une accumulation transitoire de macrophages cérébraux [34]. Son injection dans les ventricules du cerveau de rats âgés de 10 jours entraîne une forte augmentation de la concentration en ARN messagers codant pour le NGF, aussi bien dans l'hippocampe que dans le néocortex cérébral [35]. Des études menées parallèlement sur des cultures cellulaires montrent que l'interleukine 1 stimule 
la production astrocytaire de NGF ; cette stimulation est aussi observée en présence d'interleukine $6 \quad[35,36]$. Enfin, les macrophages cérébraux issus d'embryons de rat peuvent eux-mêmes produire du NGF à la suite d'une stimulation par des lipopolysaccharides. Cette propriété les distingue des phagocytes d'origine péritonéale [37].

\section{Conclusions}

L'ensemble des observations suggèrent des influences multiples des macrophages sur l'organisation des circuits neuronaux au cours de l'ontogenèse ou sur leurs remaniements dans des contextes lésionnels. La sécrétion de composés cytotoxiques et les activités de phagocytose font de ces cellules des effecteurs de régressions morphologiques ou de mort neuronale. A l'inverse, les macrophages peuvent s'intégrer dans un réseau d'interactions cellulaires qui favorise la croissance de prolongements neuronaux ou la survie de neurones. La mise en jeu de ces fonctions macrophagiques fait l'objet de contrôles à plusieurs niveaux. Elle dépend, en premier lieu, de facteurs locaux qui favorisent ou limitent le recrutement de macrophages compétents. Sans pour autant ignorer une composante d'origine vasculaire (cellules endothéliales, lymphocytes, cytokines plasmatiques...) au premier plan dans les réactions inflammatoires, il faut ici mentionner la contribution des cellules nerveuses, notamment les astrocytes capables de synthétiser des facteurs de croissance (M-CSF, GM-CSF) actifs sur les macrophages cérébraux ou leurs précurseurs [38-40]. Par ailleurs, l'expression in situ d'une fonction cytolytique ou neurotrophique relève aussi de signaux échangés entre les macrophages recrutés et les cellules nerveuses, signaux dont les composantes moléculaires restent à préciser. $\mathrm{Ce}$ nouveau domaine de recherche bénéficie a priori des données acquises sur la régulation des fonctions de

\section{Summary}

Brain macrophages: involvement in morphological changes and death of neuronal cells

Microglial cells in the developing central nervous system transiently display a macrophagic phenotype. These brain macrophages can also appear into the adult parenchyma following various tissue injuries or during neuroinflammatory and neurodegenerative diseases. Brain macrophages are involved in tissue degradation or remodeling. This paper reviews recent data related to the influence of these cells on the morphology and the survival of neurons. In vivo observations support the notion that brain macrophages can induce neuronal death or morphological regression of axonal arbors. Moreover, ex vivo cultured brain macrophages, derived from immature rat brain, produce reactive oxygen intermediates which account for deleterious effects on neurons growing in their vicinity. Conversely, studies focused on the nerve growth factor (NGF) production by nervous cells suggest mechanisms whereby brain macrophages could favour survival and growth of central neurons.

\section{Remerciements}

Les auteurs expriment leur gratitude au professeur Jacques Glowinski pour son aide permanente.

\section{TIRÉS A PART}

M. Mallat. phagocytes mononucléés dans des tis- sus non nerveux. A titre d'exemple, l'existence d'une cytotoxicité des macrophages péritonéaux induite par des neurotransmetteurs n'est pas sans intérêt pour des travaux axés sur le système nerveux central [41]
toxic effect of brain macrophage on neurons. Eur J Neurosci 1991 (sous
42. Chamak B, Mallat M. Fibronectin and of microglial cells. Neuroscience 1991 (sous presse).
43. Théry C, Chamak B, Mallat M. Cyto-

